\title{
The Effect of Daikenchuto, Japanese Herbal Medicine, on Adhesion Formation Induced by Cecum Cauterization and Cecum Abrasion in Mice
}

\author{
Songtao Wu, Naoki Uyama, Rei Atono Itou, Etsuro Hatano, Hiroko Tsutsui, and Jiro Fujimoto* \\ Department of Surgery, Division of Hepatobiliary Pancreas Surgery, Hyogo College of Medicine; 1-1 Mukogawacho, \\ Nishinomiya, Hyogo 663-8501, Japan. \\ Received July 17, 2018; accepted November 9, 2018
}

Daikenchuto (DKT) has been widely used for the treatment of postsurgical ileus in Japan. However, its effect on postsurgical adhesion formation has been obscure. In this study, the effect of DKT on postsurgical adhesion formation induced by cecum cauterization or cecum abrasion in mice was investigated. First, the expression of adhesion-related molecules in damaged ceca was investigated by quantitative (q)RT-PCR. During $24 \mathrm{~h}$ after surgery, mRNA expressions of interferon- $\gamma$ (IFN- $\gamma$ ), plasminogen activator inhibitor-1 (PAI-1), interleukin-17 (IL-17), and Substance P (SP) in cauterized ceca and those of PAI-1 and IL-17 in abraded ceca were significantly up-regulated. Next, the effect of DKT on adhesion formation macroscopically evaluated with adhesion scoring standards. DKT $(22.5-67.5 \mathrm{mg} / \mathrm{d})$ was administered orally for $7 \mathrm{~d}$ during the perioperative period, and DKT did not reduce adhesion scores in either the cauterization model (control:DKT $67.5 \mathrm{mg} / \mathrm{d}, 4.8 \pm 0.2: 4.8 \pm 0.2$ ) or in the abrasion model (control:DKT $67.5 \mathrm{mg} / \mathrm{d}, 4.9 \pm 0.1: 4.5 \pm 0.3$ ). Histologically, collagen deposition and leukocyte accumulation were found at the adhesion areas of control mice in both models, and DKT supplementation did not alleviate them. Last, effect of DKT on expression of proadhesion moleculs was evaluated. DKT also failed to down-regulate mRNA expression levels of them in damaged ceca of both models. In conclusion, PAI-1 and IL-17 may be key molecules of postsurgical adhesion formation. Collagen deposition and leukocytes accumulation are histological characteristic feature of post-surgical adhesion formation. DKT may not have any preventive effect on postsurgical adhesion formation in mice.

Key words adhesion formation; Daikenchuto; collagen deposition; leukocyte accumulation

\section{INTRODUCTION}

Postsurgical adhesions, connective tissues generated among intraperitoneal organs and tissues, occur in approximately $90 \%$ of patients undergoing abdominal surgeries. ${ }^{1,2)}$ It often results in severe complications of intestinal obstruction, female infertility, and chronic abdominal pain. ${ }^{3-5)}$ To relieve these symptoms, many patients need medications and/or reoperation, and the high cost is a heavy burden of health care. In the United States, adhesiolysis costs more than $\$ 2$ billion per year. ${ }^{6,7)}$ Because postsurgical adhesion is a significant clinical issue, the development of drugs for definitive prevention against postsurgical adhesion formation is necessary.

Mechanisms and key molecules of postsurgical adhesion formation have been reported using rodent models. Previously, we demonstrated that interferon- $\gamma($ IFN- $\gamma)$ and plasminogen activator inhibitor-1 (PAI-1) were key molecules of adhesion formation induced by cecum cauterization and by partial hepatectomy in mice. ${ }^{8,9)}$ In these models, expression of IFN- $\gamma$ and PAI-1 in cauterized ceca and remnant liver was significantly up-regulated up to $24 \mathrm{~h}$ after surgery. In both IFN- $\gamma$ knockout mice and PAI-1 knockout mice, adhesion formation was significantly inhibited compared with control mice at $7 \mathrm{~d}$ after surgery. Intriguingly, IFN- $\gamma$ knockout mice exhibited significantly impaired induction of PAI-1, indicating that IFN- $\gamma$ exerts its proadhesion action by inducing PAI-1. Interleukin-17 (IL-17) was also reported as a key molecule in formation of surgical adhesions. ${ }^{10,11)}$ In the cecum abrasion murine model, the level of IL-17 as well as IFN- $\gamma$ significantly increased in ascites up to $12 \mathrm{~h}$ after surgery, and administration of neutralizing antibodies against IL-17 or IFN- $\gamma$ significantly alleviated adhesion formation. ${ }^{11)}$ Moreover, Substance P (SP), a neurotransmitter, was also involved in postsurgical adhesion formation. ${ }^{12-14)}$ In an ischemic peritoneal injury rat model, concentrations of SP in ascites significantly increased at $6 \mathrm{~h}$ after surgery, and administration of antagonist for SP receptor significantly reduced adhesion formation. ${ }^{14,15)}$ Notably, expression of the proadhesion molecules mentioned above increased in the early phase, up to $24 \mathrm{~h}$ after surgery.

Clinically, Daikenchuto (DKT), a Japanese Kampo herb, is often used for the treatment of postsurgical ileus and is reported to shorten the duration of hospital stay of patients with postsurgical ileus. ${ }^{16)}$ Postsurgical ileus has been thought to occur due to delayed intestinal transit and inflammation. ${ }^{17)}$ DKT treatment has been reported to improve intestinal motility. In conscious dogs and mice with postsurgical ileus, DKT treatment rescued delayed intestinal transit through activation of cholinergic efferent neurons. ${ }^{18,19)}$ Acetylcholine, their transmitter, is well established to be capable of inhibiting inflammation via stimulation of the nicotinic acetylcholine receptor (nAchR) on leukocytes. ${ }^{20,21)}$ Therefore, it is plausible that DKT treatment reduces enteric neural inflammation by inhibiting the infiltration of leukocytes through nAchR, which eventually results in further improvement of intestinal motility. ${ }^{19)}$ This may partly underlie DKT's effect on the reduction of symptoms of postsurgical ileus. Furthermore, DKT was demonstrated to reduce nonsurgical, talc-induced intestinal adhesion formation by activating transient receptor potential 
vanilloid type-1 (TRPV-1), a cationic channel preferentially expressed on alimentary afferent neurons. ${ }^{22)}$ Recently, DKT was shown to act on a type of $\mathrm{Ca}^{2+}$ channel and potassium leak channels in the gut as well. ${ }^{23)}$ However, it remains to be elucidated whether DKT can protect against postsurgical adhesion formation.

In the present study, we investigated whether DKT prevented postsurgical adhesion formation induced by 2 types of surgeries, cecum cauterization and cecum abrasion, particularly focusing on the effects of DKT on the induction of the proadhesion molecules IFN- $\gamma$, PAI-1, IL-17, and SP.

\section{MATERIALS AND METHODS}

Animal Experiment Female BALB/c mice (10-11 weeks of age, $25 \mathrm{~g}$ body weight) were purchased from SLC (Hamamatsu, Japan). The study was approved by the Institutional Animal Care Committee, Hyogo College of Medicine.

Surgical Procedures of the Cecum Cauterization Model and Cecum Abrasion Model Two surgical adhesion mouse models, cecum cauterization and cecum abrasion, were used for the present study. A $10-\mathrm{mm}$ midline incision was made in the abdominal wall of mice in both models under anesthesia with $3 \%$ isoflurane. In the cauterization model, the cecum was isolated and cauterized using the coagulation mode of bipolar forceps for $1 \mathrm{~s}\left(30 \mathrm{~W}, 500 \mathrm{kHz}\right.$; MERA, Tokyo, Japan). ${ }^{8)}$ In the abrasion model, the cecum was abraded 3 times with a cotton swab, leading to visible damage, and the opposing area of the abdominal wall was also abraded 3 times using appropriate force. ${ }^{10,24)}$ After treatment, the cecum was restored to its anatomical place in the abdominal cavity, and the abdominal wall was closed in 2 layers using a 5-0 silk suture in a running fashion in both models.

Quantitative (q)RT-PCR) At 0, 3, 6, 12, and 24h after surgery, mice were sacrificed (Fig. $1 \mathrm{~B}$ and Fig. 5A). Total mRNA was extracted from the damaged ceca using the RNeasy Mini Kit (Qiagen, Valencia, CA, U.S.A.) and the cDNA was synthesized using Superscript III Reverse Transcriptase (Thermo Fisher Scientific, Waltham, MA, U.S.A.). The expression of the adhesion-related molecules at the mRNA level was determined by qRT-PCR. PCR primers with TaqMan probe for $18 \mathrm{~S}$ ribosomal RNA (rRNA) (Hs99999901_s1), IFN- $\gamma$ (Mm01168134_m1), PAI-1 (Mm00435858_m1), IL-17 (Mm00439618_m1), and Substance P (Mm01166996_ml) and TaqMan Universal PCR Master Mix were from Applied Biosystems (Foster City, CA, U.S.A.). The fold change of each gene expression was analysed using the $2^{-\Delta \Delta \mathrm{Ct}}$ method. ${ }^{25)}$ According to the $2^{-\Delta \Delta \mathrm{Ct}}$ method, each $\mathrm{Ct}$ value was first normalized to the internal reference gene (18S) of the sample and, afterward, to the controls.

Administration of DKT and Macroscopic Evaluation of Adhesion Formation DKT extract powder and maltose syrup powder were gifts from Tsumura \& Co. (Tokyo, Japan). DKT extract was made from a crude drug mixture of processed ginger, ginseng, and zanthoxylum fruit, at a ratio of $5: 3: 2$. DKT extract powder and maltose syrup powder were mixed at a ratio of $1: 8$ and used as DKT. In each adhesion mouse model, 32 mice were divided into 4 groups $(n=8 /$ group): control group (administration of distilled water), DKT1 group (administration of $22.5 \mathrm{mg} /$ mouse DKT), DKT2 group (administration of $45.0 \mathrm{mg} /$ mouse DKT), and DKT3 group (administration of $67.5 \mathrm{mg} /$ mouse DKT). According to the reports, we decided to administer DKT by these three dosages. $^{22,26,27)}$ Just before administration, DKT was dissolved in $250 \mu \mathrm{L}$ distilled water, and the DKT solution was given orally to mice once a day with animal-feeding needles (diameter $\times$ length: $0.9 \times 50 \mathrm{~mm} ; \mathrm{KN}-348$, Natsume, Tokyo, Japan). In the cecum cauterization model, the DKT solution was administered for 7 consecutive days beginning $1 \mathrm{~d}$ before surgery (Fig. 2A), whereas in the cecum abrasion model, it was administered for 7 consecutive days beginning $5 \mathrm{~d}$ before surgery (Fig. 6A). In the control group of both models, an equivalent volume of distilled water $(250 \mu \mathrm{L} /$ mouse) was administered. The adhesion formation in the abdominal cavity was evaluated macroscopically $7 \mathrm{~d}$ after the surgery using adhesion scoring standards. ${ }^{8)}$ Briefly, the adhesion scoring standards are as follows: 0, without any adhesion; 1, single thin, filmy adhesion; 2, two or more thin adhesion or moderate adhesion; 3, moderate adhesion with focal point; 4, thick adhesion with plantar attachment or multiple thick adhesions with focal point; 5, thick, wide vascularized adhesion.

Mallory-Azan Staining and Immunohistochemistry Tissues of adhesion formation at $7 \mathrm{~d}$ after surgery were fixed for $48 \mathrm{~h}$ at room temperature with $10 \%$ formalin. The tissues were dehydrated with ethanol and xylene and embedded in paraffin wax. Sections with adhesion tissue $4-\mu \mathrm{m}$ thick were placed on hydrophilic silanized slides (DAKO, Carpinteria, CA, U.S.A.), incubated for $30 \mathrm{~min}$ at $63^{\circ} \mathrm{C}$, deparaffinized with xylene, and rehydrated with ethanol. Antigen was retrieved by incubating for $40 \mathrm{~min}$ at $95^{\circ} \mathrm{C}$ in $10 \mathrm{mM}$ citrate buffer $(\mathrm{pH}$ 6.0). Mallory-Azan staining was performed for detection of collagen deposition with an azocarmine $\mathrm{G}$ aqueous solution, a 5\% molybdenum phosphate aqueous solution and an aniline blue and Orange G-mixed aqueous solution. As for immunostaining for collagen type I and CD45, a marker for leukocytes, the slides were incubated in a $0.3 \%$ solution of $\mathrm{H}_{2} \mathrm{O}_{2}$ in absolute methanol for $30 \mathrm{~min}$ at room temperature for quenching endogenous peroxidase. After blocking with Protein Block Serum-Free (DAKO) for $30 \mathrm{~min}$ at room temperature, the slides were incubated overnight at $4^{\circ} \mathrm{C}$ with rabbit anti-collagen type I primary antibody (Bio-Rad, Richmond, CA, U.S.A.) at a dilution of 1/500 in phosphate-buffered saline (PBS) and rat anti-CD45 primary antibody (Clone: 30-F11; BD Biosciences, San Jose, CA, U.S.A.) at a dilution of $1 / 200$ in PBS, respectively. The second antibody staining was performed by incubating for $30 \mathrm{~min}$ at room temperature with peroxidase-labeled secondary antibodies, Hitofine Simple Stain Mouse Max PO (R) (Nichirei Biosciences, Tokyo, Japan) and Hitofine Simple Stain Mouse Max PO (MULTI) (Nichirei), respectively. The slides were incubated with 3,3'-diaminobenzidine- $\mathrm{H}_{2} \mathrm{O}_{2}$ for 3 min, rinsed with tap water, counterstained with Mayer's hematoxylin, and rinsed with tap water again. They were dehydrated with methanol and xylene and mounted with Malinol. The staining was visualized under a Nikon Eclipse TS-100 microscope (Nikon, Tokyo, Japan).

Statistical Analysis All data are presented as means \pm standard error of the mean (S.E.M.). The differences between the groups were evaluated statistically using Student $t$-test (IBM SPSS Statistics software, version 21.0, IBM, New York, NY, U.S.A.), and $p$ value less than 0.05 was considered significant. All data were compared to control groups. 
A

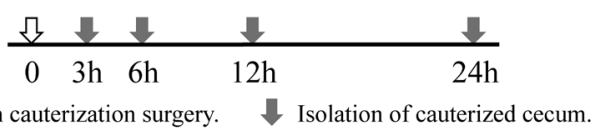

B
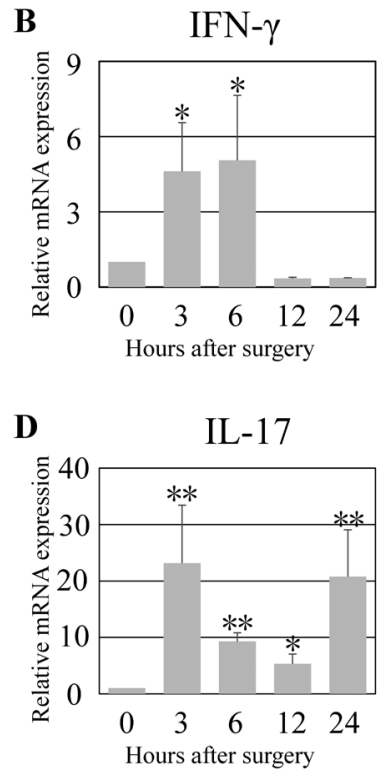

C

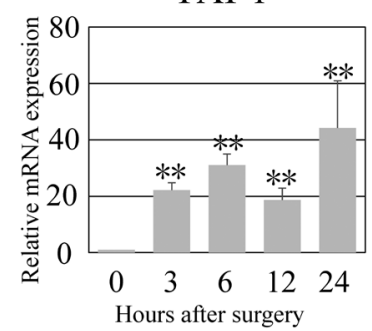

$\mathbf{E}$

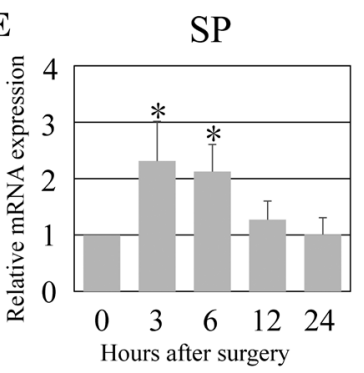

Fig. 1. Time Course of mRNA Expression Levels of Adhesion-Related Molecules in Cauterized Ceca

Cauterized ceca were isolated at $3,6,12$, and $24 \mathrm{~h}$ after surgery (A). The cecum of mice without treatment was used as control. The mRNA expression levels of IFN- $\gamma$ (B), PAI-1 (C), IL-17 (D), and SP (E) were measured by qRT-PCR. Data are presented as the mean \pm S.E.M. ( $n=5 /$ group). Expressions of all adhesion-related genes significantly increased during $24 \mathrm{~h}$ after cecum cauterization. $* p<0.05$; $* * p<0.01$.

\section{RESULTS}

Induction of IFN- $\gamma$, PAI-1, IL-17, and SP in Cauterized Ceca We substantiated further kinetics of cecal induction of proadhesion molecules in the early phase after cecum cauterization (Fig. 1A). Consistent with our previous report, ${ }^{8)}$ mRNA expression levels of IFN- $\gamma$ in the cauterized ceca were significantly up-regulated to about 5-fold of those of control mice without surgical manipulation at 3 and $6 \mathrm{~h}$ after surgery, which was followed by prompt reduction to less than those of control mice at $12 \mathrm{~h}$ and thereafter (Fig. 1B). mRNA expression levels of PAI-1 and IL-17 in the cauterized ceca significantly increased to about 20 -fold of those of control mice at $3 \mathrm{~h}$ after surgery. These high expression levels were sustained but with some fluctuations for up to $24 \mathrm{~h}$ (Figs. 1C, D). Similar to the kinetics of IFN- $\gamma$, mRNA expression levels of SP were elevated to about twice those of control mice at 3 and $6 \mathrm{~h}$ after surgery, followed by their decrease to control levels at $12 \mathrm{~h}$ (Fig. 1E). Thus, mRNA expression levels of IFN- $\gamma$, PAI-1, IL-17, and SP in the cauterized cecum were significantly upregulated with kinetics proper to the individual molecules in the early phase postsurgery.

DKT Failed to Prevent Cecum Cauterization-Induced Adhesion Formation Next, we investigated the effect of DKT on adhesion formation induced by cecum cauterization. Three DKT administration groups (DKT1 group: $22.5 \mathrm{mg}$ / mouse, DKT2 group: $45 \mathrm{mg} /$ mouse, and DKT3 group: $67.5 \mathrm{mg} / \mathrm{mouse})$ and a control group $(250 \mu \mathrm{L}$ distilled water $)$ were prepared. DKT solution or distilled water was admin-

$\mathbf{A}$

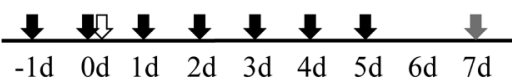

$\checkmark$ Oral administration of distilled water or DKT for 7 days.

$\Omega$ Cecum cauterization surgery.

$\checkmark$ Sacrifice, and macroscopical and histological examination.

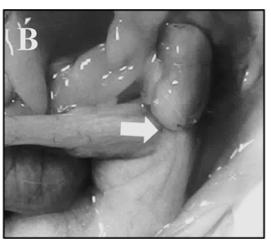

Control (Distilled water)

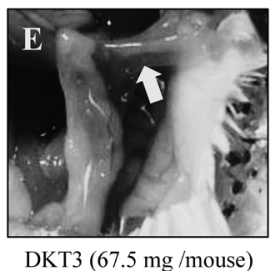

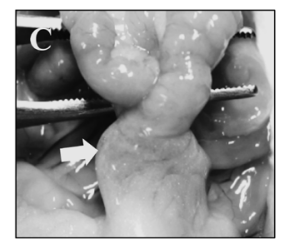

DKT1 (22.5 mg /mouse)
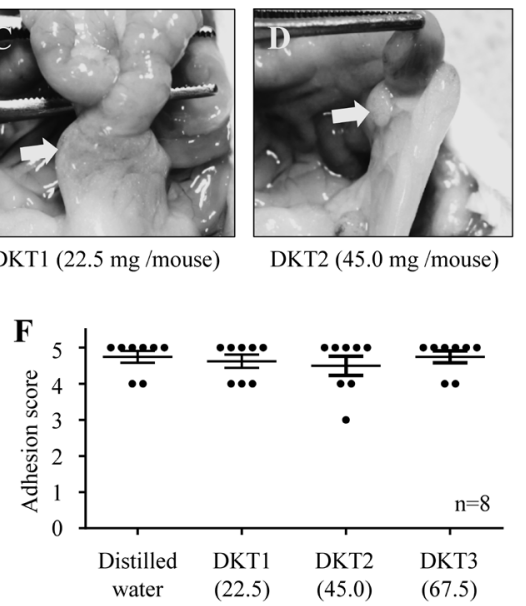

Fig. 2. Effect of DKT on Adhesion Formation in the Cecum Cauterization Model

Distilled water $(250 \mu \mathrm{L})$ or DKT solution $(250 \mu \mathrm{L})$ was daily administered orally for $7 \mathrm{~d}$ beginning $1 \mathrm{~d}$ before surgery (A). Mice were divided into 4 groups: control group (distilled water only, $n=8)(\mathrm{B})$, DKT1 group $(22.5 \mathrm{mg} / \mathrm{mouse}, n=8)(\mathrm{C})$, DKT2 group ( $45.0 \mathrm{mg} /$ mouse, $n=8)(\mathrm{D})$, and DKT3 group $(67.5 \mathrm{mg} / \mathrm{mouse}, n=8)$ (E). At $7 \mathrm{~d}$ after surgery, mice were sacrificed and adhesion formation (white arrows) was macroscopically evaluated according to adhesion scoring standards. Representative photos are shown (B-E). (F). No significant inhibitory effect of DKT on adhesion formation was found.

istered for 7 consecutive days beginning $1 \mathrm{~d}$ before surgery (Fig. 2A). At $7 \mathrm{~d}$ after surgery, severe adhesion formation was found in the control group (Fig. $2 \mathrm{~B}$; adhesion score: $4.8 \pm 0.2$ ). Severe adhesion formations comparable with the control group were observed in all DKT groups (Figs. 2C, D, E). Adhesion scores of the DKT1, DKT2, and DKT3 groups were $4.6 \pm 0.2$, $4.5 \pm 0.3$, and $4.8 \pm 0.2$, respectively. There was no significant difference in adhesion scores between the control group and each DKT group (Fig. 2F).

To evaluate the histological difference between the control group and DKT group, Mallory-Azan staining and immunostainings for collagen type I for fibrosis and for leukocyte marker CD45 for inflammation were performed. In the control group, collagen deposition (Figs. 3A, C, E) was found especially at the adhesion area of both cauterized cecum (Fig. $3 \mathrm{~A}$, arrows) and fatty tissues adhered to it. Leukocyte accumulation (Fig. 3G, arrowheads) was found in the damaged cecal wall and in the adhered fatty tissues, especially in the nonadhesion sites as well as in the adhered area, suggesting that leucocytes might be recruited not only from cecum but also from adhered tissues. In the DKT3 group, comparable collagen deposition (Figs. 3B, D, F) was found at the adhesion area of fatty tissues and damaged ceca (Fig. 3B, arrow), with comparable leukocyte accumulation (Fig. 3H, arrowheads) in the wall of cauterized cecum and adhered fatty tissues. Compared with the control group, an apparent reduction in collagen deposition or leukocyte accumulation was not found in the DKT3 group. These data indicate that DKT administration did not inhibit adhesion formation induced by cecum cauterization. 

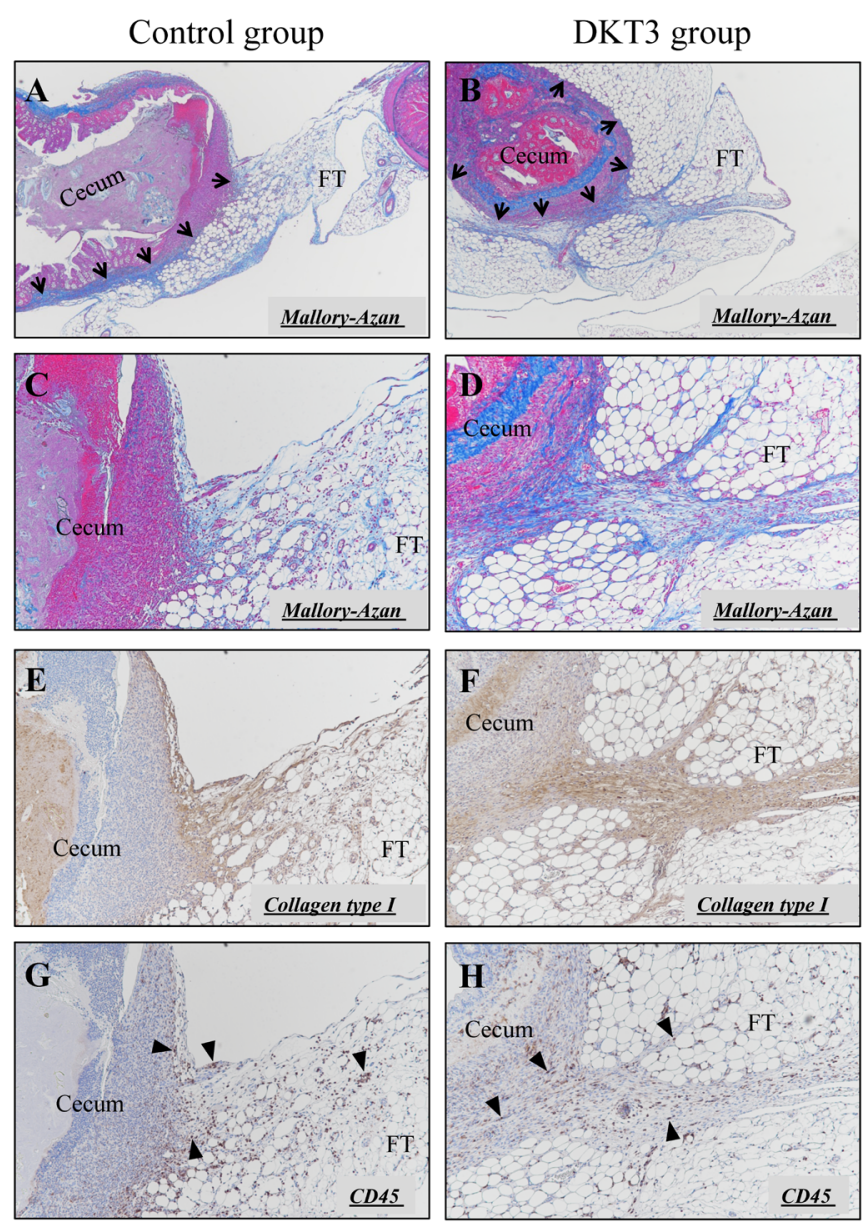

Fig. 3. Collagen Deposition and Leukocyte Accumulation in the Cauterized Ceca in DKT-Treated Groups

Collagen deposition was evaluated by Mallory-Azan staining (A, B, C, and D) and immunostaining for collagen type I (E and F), and leukocyte accumulation was evaluated by immunostaining for CD45 ( $\mathrm{G}$ and $\mathrm{H})$. Adhesion of damaged cecum and fatty tissues was found in the control group (A, black arrows) and DKT-treated groups (DKT3 group: B, black arrows). Collagen was deposited in the adhesion areas of damaged cecum and fatty tissues in both groups (control group: A, C, and E; DKT3 group: B, D, and F). In serial sections, leukocyte accumulation (G and $\mathrm{H}$, arrowheads) was found in the wall of damaged cecum and the nonadhering site of fatty tissues in addition to the adhesion area in both groups (control group: G; DKT3 group: H). Representative data are shown. No inhibitory effect of DKT on collagen deposition and leukocyte accumulation was found. FT, fatty tissues. Magnification is $\times 40$ for $\mathrm{A}$ and $\mathrm{B}, \times 100$ for $\mathrm{C}-\mathrm{H}$. (Color figure can be accessed in the online version.)

DKT Failed to Inhibit Induction of Adhesion-Related Gene Expressions The expression of adhesion-related genes in cauterized ceca of control and DKT3 groups was investigated at $6 \mathrm{~h}$ after surgery (Fig. 4A). There were no significant differences in expression levels of IFN- $\gamma$ (Fig. 4B), PAI-1 (Fig. 4C), IL-17 (Fig. 4D), or SP (Fig. 4E) between these 2 groups. These data are consistent with the comparable macroscopic and histological alterations in the DKT group (Figs. 2, 3). Furthermore, we investigated the effect of DKT administration for 7 consecutive days before surgery on the expression of adhesion-related genes in cauterized ceca of control and DKT3 group at $6 \mathrm{~h}$ after surgery (sFig. 1A). Similar to the result of 1-d administration of DKT, no significant differences were found in the expression levels of IFN- $\gamma$ (sFig. 1B), PAI-1 (sFig. 1C), IL-17 (sFig. 1D), or SP (sFig. 1E) between these two groups.

PAI-1 and IL-17, but Not IFN- $\gamma$ or SP, Were Induced after Cecum Abrasion Surgery We investigated the kinet-
A

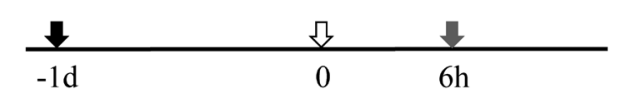

7 Oral administration of distilled water or DKT (67.5 mg /mouse).

Љ Cecum cauterization surgery.

$\checkmark$ Isolation of cauterized cecum.
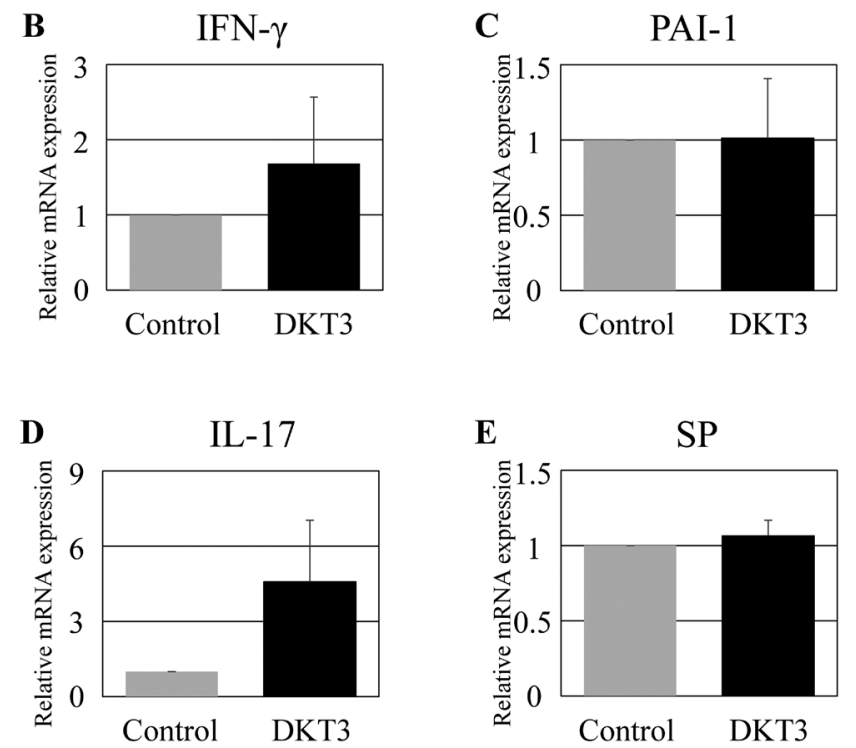

Fig. 4. Effect of DKT on the Adhesion-Related Molecules Expression in the Cecum Cauterization Model

Distilled water $(250 \mu \mathrm{L}$ : control group, $n=5)$ or DKT solution (DKT3 group: $67.5 \mathrm{mg}$ DKT/mouse, $n=5$ ) was administered orally $1 \mathrm{~d}$ before surgery (A). Expression of IFN- $\gamma$ (B), PAI-1 (C), IL-17 (D), and SP (E) was measured by qRT-PCR at $6 \mathrm{~h}$ after surgery, and gene expressions of these molecules between the control group and DKT3 group were compared. DKT did not significantly inhibit the expression of all adhesion-related molecules.

ics of adhesion-related gene induction up to $24 \mathrm{~h}$ after cecum abrasion surgery (Fig. 5A). In contrast to the cecum cauterization model, mRNA expression levels of IFN- $\gamma$ were not significantly up-regulated in the abraded ceca up to $24 \mathrm{~h}$ after surgery (Fig. 5B). As in the case of cecum cauterization, expression levels of PAI-1 significantly increased approximately to 20 -fold of controls at $3 \mathrm{~h}$ after surgery and were sustained until $24 \mathrm{~h}$ after surgery (Fig. 5C). mRNA expression levels of IL-17 were gradually and significantly up-regulated to 10 -fold that of controls at $24 \mathrm{~h}$ after surgery (Fig. 5D). Like IFN- $\gamma$, mRNA expression levels of SP were not significantly elevated (Fig. 5E). Thus, gene expressions of PAI-1 and IL-17, but not IFN- $\gamma$ or SP, were significantly induced in the abraded ceca.

Adhesion Formation Induced by Cecum Abrasion Was Not Inhibited by DKT Next, we investigated the effect of DKT on adhesion formation induced by cecum abrasion (Fig. $6 \mathrm{~A}$ ). As in the cecum cauterization model, severe and comparable adhesion formation was found in both the control group (Fig. 6B) and the DKT-administered groups (Figs. 6C, D, E). No differences were found in adhesion score between the control group and each DKT-treated group (control group:DKT1 group:DKT2 group:DKT3 group, 4.9 $\pm 0.1: 4.8 \pm 0.2: 4.6 \pm$ $0.2: 4.5 \pm 0.3$; Fig. $6 \mathrm{~F}$ ). This is also the case for histological changes in terms of fibrosis and inflammation. In the control group, collagen deposition (Figs. 7A, C, E) was found at the adhesion area of fatty tissues, neighbor intestine, and damaged cecum (Fig. 7A, black arrows). Leukocyte accumulation 
A

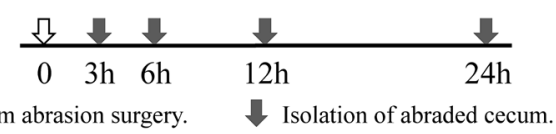

B
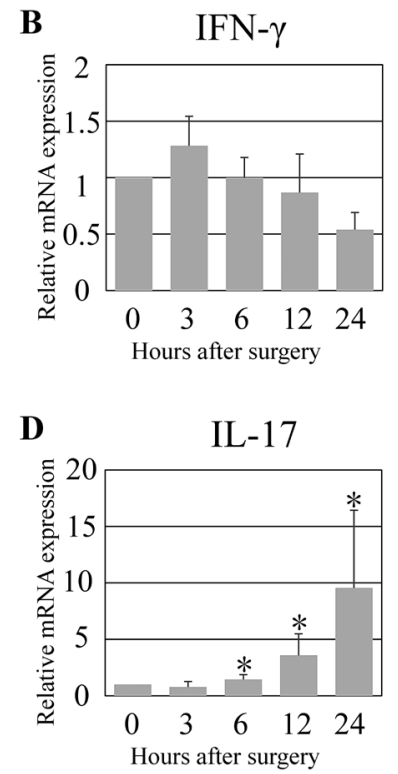

C

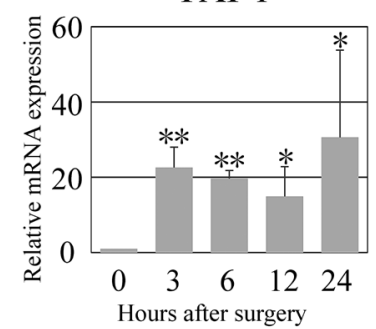

$\mathbf{E}$

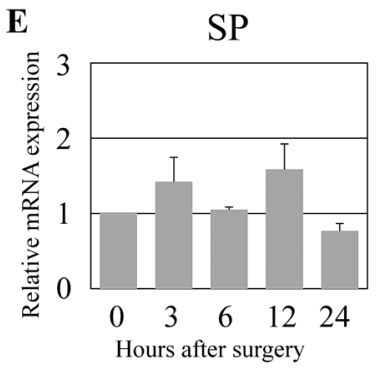

Fig. 5. Time Course of Expression of Adhesion-Related Molecules in Abraded Ceca at the mRNA Levels

Abraded ceca were isolated at 3, 6, 12, and $24 \mathrm{~h}$ after surgery (A). Cecum without treatment was used as a control. The expressions of IFN- $\gamma$ (B), PAI-1 (C), IL-17 (D), and SP (E) were measured by qRT-PCR. Data are presented as the mean \pm S.E.M. ( $n=5 /$ group). Expression of PAI-1 (C) and IL-17 (D) was significantly up-regulated for $24 \mathrm{~h}$ after cecum abrasion. $* p<0.05 ; * * p<0.01$.

(Fig. 7G, arrowheads) was found in the damaged ceca and the adhered fatty tissues, even at their nonadhesion sites. In the DKT3 group, adhesion was observed in fatty tissues, neighbor intestine, and damaged ceca (Fig. 7B, arrows). Collagen deposition was found around the adhesion area as well (Figs. 7D, F). Leukocyte accumulation was found in the wall of damaged ceca and the adhered fatty tissues, in both adhered and nonadhered sites (Fig. 7H, arrowheads). Compared with the control group, no apparent reduction in fibrosis or inflammation was found in the DKT3 group. These data indicate that DKT administration did not inhibit adhesion formation induced by cecum abrasion.

Early Induction of Adhesion-Related Genes Was not Dampened by DKT Treatment Expression of adhesionrelated genes in the ceca of control mice and DKT-treated mice (DKT3 group) at $6 \mathrm{~h}$ after cecum abrasion was also investigated by qRT-PCR (Fig. 8A). In the DKT3 group, induction of PAI-1 and SP was not significantly reduced as compared with the control group (Figs. 8C, E). Intriguingly, in the DKT3 group, IFN- $\gamma$ and IL-17 levels were significantly higher, respectively, than those in the control group (Figs. 8B, D). However, macroscopical or histological severe changes in the DKT group were not obviously observed (Figs. 6, 7).

\section{DISCUSSION}

The present study reveals that DKT supplementation cannot improve postsurgical adhesion formation. Indeed, DKT could not protect against adhesion formation induced by cecum cauterization or cecum abrasion, in terms of clinical scores,

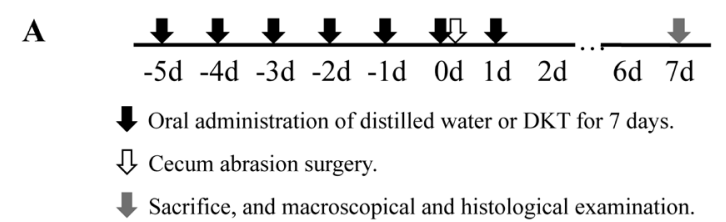

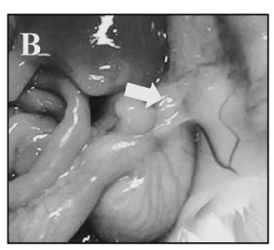

Control (Distilled water)

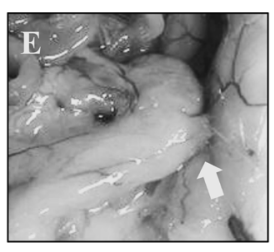

DKT3 (67.5 mg /mouse)

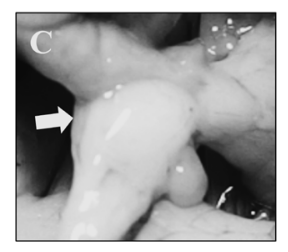

DKT1 (22.5 mg /mouse)

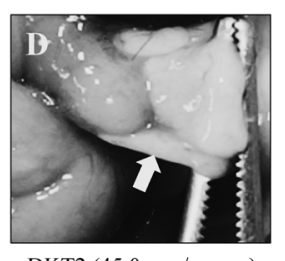

DKT2 (45.0 mg /mouse)

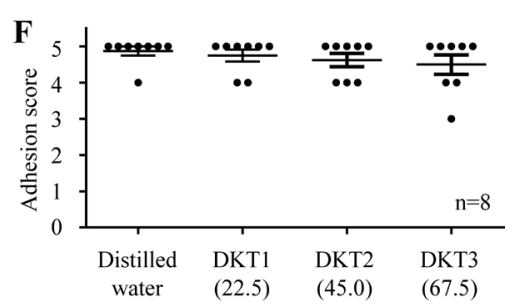

Fig. 6. Effect of DKT on Adhesion Formation in the Cecum Abrasion Model

Distilled water $(250 \mu \mathrm{L})$ or DKT solution $(250 \mu \mathrm{L})$ was administered orally for $7 \mathrm{~d}$ beginning $1 \mathrm{~d}$ before surgery (A). Mice were divided into 4 groups: control group (distilled water only, $n=8)$ (B), DKT1 group $(22.5 \mathrm{mg} / \mathrm{mouse}, n=8)(\mathrm{C})$, DKT2 group $(45.0 \mathrm{mg} / \mathrm{mouse}, n=8)(\mathrm{D})$, and DKT3 group $(67.5 \mathrm{mg} / \mathrm{mouse}, n=8)(\mathrm{E})$. Adhesion formation (white arrows) was macroscopically evaluated at $7 \mathrm{~d}$ after surgery by adhesion scoring standards. Representative data are shown. No significant inhibitory effect of DKT on adhesion formation was found between the control group and DKT-treated groups (E).

histological changes, and induction of proadhesion molecules in the damaged cecum. Previous reports demonstrated that DKT supplementation is beneficial for protection against postsurgical adverse events through activating neural fibers. First, DKT activates intestinal cholinergic efferent neurons to release acetylcholine, which in turn stimulates nAchR on leukocytes to dampen intestinal nerve inflammation relevant to intestinal motility failure. ${ }^{19)}$ This anti-ileus action of DKT seems insufficient to protect against postsurgical adhesion formation, at least those in 2 types of mouse models, as shown here. Second, DKT or DKT components can prevent adhesion formation induced by nonsurgical manipulation via activating the chemo- and thermal sensor, TRPV-1.22) Other ion channels expressed in the gut have been demonstrated to be targeted for the beneficial action of DKT. ${ }^{23)}$ Even in the presence of those beneficial neural and enterocytic responses to administration of DKT, adhesion formation normally develops upon surgical manipulation, as shown in this study. ${ }^{20-23,28,29)}$ For protection against postsurgical adhesion formation, medication targeting the other pathways might be required.

PAI-1 is an inhibitor of plasminogen activator (PA), an enzyme that converts zymogen plasminogen into enzymatically active plasmin, which is responsible for fibrinolysis by degradation of fibrin. PAI-1 is expressed by various cell types, such as neutrophils, macrophages, fibroblasts, endothelial cells, and mesothelial cells. ${ }^{30-32)}$ PAI-1 is considered to stabilize the fibrin matrix, a provisional matrix for migration and proliferation of fibroblasts, by inhibiting the activity of PA. ${ }^{33,34)}$ In addition, PAI-1 has been reported to be deeply involved in tissue fibrosis. ${ }^{31,32)}$ For example, liver fibrosis induced by 
Control group
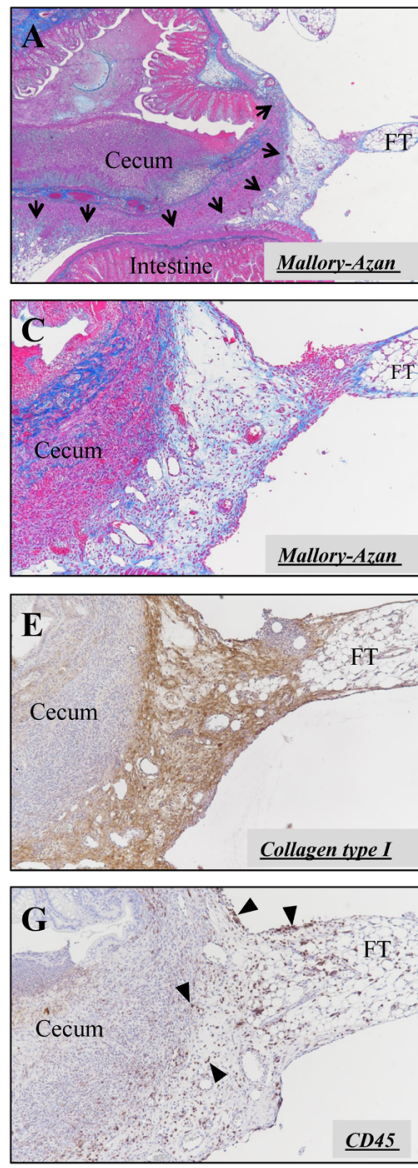

DKT3 group
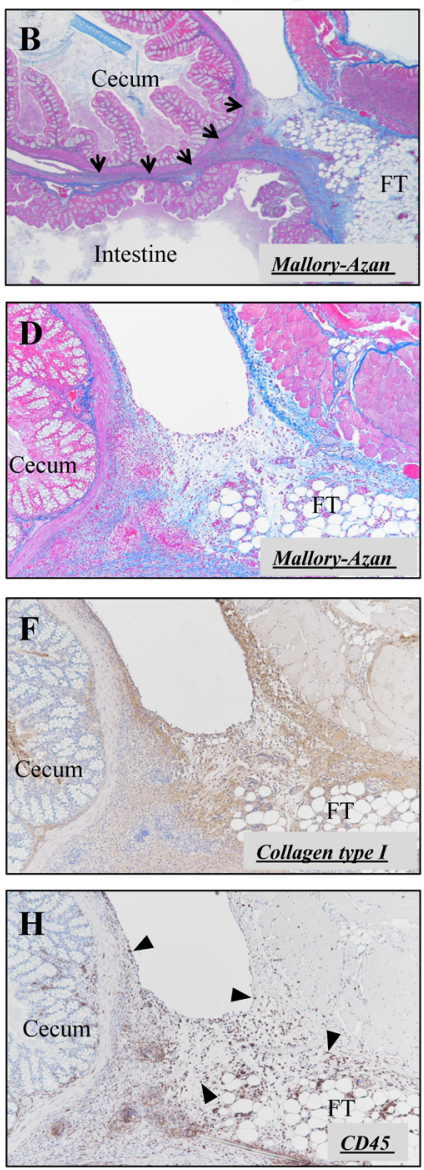

Fig. 7. Collagen Deposition and Leukocyte Accumulation in the Control Group and DKT-Treated Groups of the Cecum Abrasion Model

Collagen deposition was evaluated by Mallory-Azan staining (A, B, C, and D) and immunostaining for collagen type $\mathrm{I}$ ( $\mathrm{E}$ and $\mathrm{F}$ ), and leukocyte accumulation was evaluated by immunostaining for $\mathrm{CD} 45(\mathrm{G}$ and $\mathrm{H})$. The adhesion area of the damaged cecum and fatty tissues was found in the control group (A, black arrows) and DKT-treated groups (DKT3 group: B, black arrows). Collagen was deposited in the adhesion area of the damaged cecum and fatty tissues in both groups (control group: A, C, and E; DKT3 group: B, D, and F). In serial sections, leukocyte accumulation ( $\mathrm{G}$ and $\mathrm{H}$, arrowheads) was found in the wall of damaged cecum and adhering fatty tissues in addition to the adhesion area in both groups (control group: G; DKT3 group: H). Representative data are shown. No inhibitory effect of DKT on collagen deposition and leukocyte accumulation was found. FT, fatty tissues. Magnification is $\times 40$ for $\mathrm{A}$ and $\mathrm{B}, \times 100$ for $\mathrm{C}-\mathrm{H}$. (Color figure can be accessed in the online version.)

bile duct ligation was attenuated in PAI-1 knockout mice. In the absence of PAI-1, PA was sustained to be enzymatically active, which results in persistent production of plasmin, eventually leading to the aberrant production of plasmin-sensitive, antifibrotic substrates, such as biologically active hepatocyte growth factor and matrix metalloproteinases-9. ${ }^{35)}$ This suggests that PAI-1 is involved in liver fibrosis as an inhibitor of fibrinolysis. Intriguingly, PAI-1 reportedly exerts its action independently of PA or plasmin as well. Renal fibrosis induced by unilateral ureteral obstruction was ameliorated in PAI-1 knockout mice. In this case, the absence of PAI-1 reduces transforming growth factor- $\beta 1$ (TGF- $\beta 1$ ) expression in renal tissue without elevation of PA explosion levels or plasmin activity. ${ }^{36)}$ These articles indicate that PAI-1 exerts its profibrotic action depending on or independently of its fibrinolysis-inhibiting action. Previously, we reported that adhesion formation induced by partial hepatectomy was ameliorated in PAI-1-depleted mice. ${ }^{9)}$ Thus, PAI-1 is an essential factor for

A

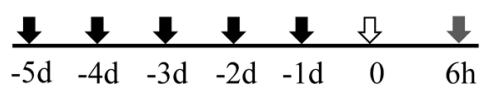

$\downarrow$ Oral administration of distilled water or DKT (67.5 mg /mouse).

\& Cecum abrasion surgery.

$\checkmark$ Isolation of abraded cecum.
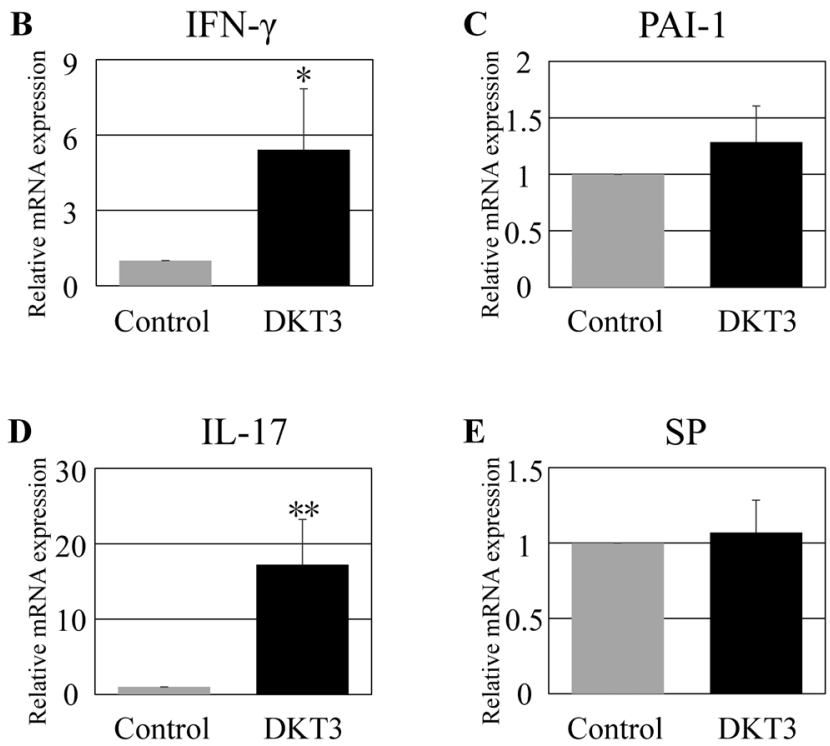

Fig. 8. Effect of DKT on the Adhesion-Related Molecules Expression at 6 hours after Surgery in the Cecum Abrasion Model

Distilled water $(250 \mu \mathrm{L}$ : control group, $n=5)$ or DKT solution (DKT3 group: $67.5 \mathrm{mg}$ DKT $/$ mouse, $n=5$ ) was administered orally for $5 \mathrm{~d}$ before surgery (A). Abraded ceca were isolated at $6 \mathrm{~h}$ after surgery. The expression of IFN- $\gamma$ (B), PAI-1 (C), IL-17 (D), and SP (E) was investigated by qRT-PCR, and the expression of these molecules was compared between the control group and DKT3 group. DKT did not significantly inhibit the expression of adhesion-related molecules.

\section{collagen production in adhesion formation.}

IL-17 is produced by a variety of cells, such as neutrophils, $\mathrm{CD}^{+} \mathrm{T}$ cells, $\gamma \delta \mathrm{T}$ cells, natural killer cells, macrophages, natural killer $\mathrm{T}$ cells, and dendritic cells. ${ }^{37,38)}$ Since IL-17 is essential for the recruitment and activation of neutrophils, it is plausible that IL-17 is indispensable for host defense against microbial infection. ${ }^{39,40)}$ Furthermore, IL-17 has been shown to be important for development of tissue fibrosis. Depletion of neutrophils or blockade of IL-17 significantly decreases pulmonary and renal fibrosis. ${ }^{41,42)}$ Thus, neutrophil-derived IL-17 seems to be indispensable for certain types of tissue fibrosis. Blockade of IL-17 significantly inhibited adhesion formation induced by cecum abrasion, although the cellular source of IL-17 remains to be elucidated. ${ }^{43)}$

Collagen type I induction is well established as an important event for adhesion formation after surgery of peritoneal patch. ${ }^{44)}$ The protective effect of DKT administration on collagen type 1 production was reported in a rat colonic fibrosis model and a mouse peritoneal fibrosis model. ${ }^{26,45)}$ In a rat colonic fibrosis model, DKT administration prevents trinitrobenzen sulfonic acid (TNBS)-induced mucosal fibrosis, with reduction in induction of mRNA expressions of TGF- $\beta$ and collagen type 1 . In a mouse peritoneal fibrosis model, DKT administration rescues chlorhexidine gluconate-induced peritoneal fibrosis, concomitant with reduced induction of $\alpha$ smooth muscle actin ( $\alpha$ SMA), a marker of collagen-producing myofibroblasts, and of phosphorylated $\operatorname{smad} 2 / 3$, a signature 
of TGF- $\beta$ signaling. DKT also reduced accumulation of macrophages with dampened induction of macrophage-recruiting chemokine, MCP1. Intriguingly, DKT treatment commonly diminishes induction of heat shock protein (HSP)-47, which is a collagen-specific molecular chaperone and is essentially required for processing of procollagen into collagens. ${ }^{46,47)}$ Thus, the DKT protection against tissue fibrosis might be attributable to reduced induction of HSP-47. In contrast, DKT treatment did not down-regulate collagen type 1 induction in the injured site after cecal cauterization and ablation. This may suggest that DKT reduction of HSP-47 induction might be insufficient for protection against adhesion formation in our models. Further study is needed.

The cell origin of myofibroblasts that produces collagen type $I$ in postsurgical adhesion remains unclear. Peritoneal fibroblasts, mesothelial cells, and submesothelial fibroblasts are thought to be candidates. ${ }^{48-50)}$ Further studies are necessary to determine the cells responsible for collagen production.

DKT administration was reported to alleviate TNBSinduced colitis and fibrosis, with down-regulating IFN- $\gamma$ induction in damaged tissue. ${ }^{27)}$ Intriguingly, DKT treatment induces production of adrenomodulin (ADM), which is a potent anti-inflammatory peptide, as well. Since administration of ADM antagonist diminishes the protective effects of DKT, DKT exerts its anti-colitis and -Sadhesion actions via inducing ADM. Indeed, Kono et al. showed that ADM reduced IFN- $\gamma$ production by $\mathrm{T}$ cells activated with $\mathrm{T}$ cell receptor engagement. ${ }^{27)}$ By contrast, in our models, DKT administration did not reduce induction of mRNA expression of IFN- $\gamma$. As previously reported, blockade of IFN- $\gamma$ prevented adhesion formation in a cecum cauterization mouse model. ${ }^{8)} \mathrm{We}$ found that serosal resident NKT cells, but not T cells, produced IFN- $\gamma$ in response to neuropeptide substance $\mathrm{P}$, which might be promptly released in the injured serosa. ${ }^{8}$ There are several reasons for the different effects of AMD induced by DKT. First, AMD produced in the mucosal regions might be consumed or diminished to the levels insufficient to exert its suppressive action, during spreading to the serosa. Second, NKT cells might not express ADM receptor. Third, ADM might lack inhibiting action onto substance P-mediated IFN- $\gamma$ production.

IFN- $\gamma$ levels along with IL-17 levels were reported to be elevated in the early phase after cecal abrasion. ${ }^{11)}$ However, the present study showed a lack of significant induction of IFN- $\gamma$ after cecal abrasion. This discrepancy might be accounted for by different methods of abrading manipulation.

Expression of IL-17 was significantly up-regulated in the abraded ceca of DKT-treated mice without the apparently severe induction of adhesion intensity compared with control mice. This may implicate that IL-17 exerts its pro-inflammatory action in a step-by-step manner. Although the detailed mechanism of DKT induction of IL-17 needs to be elucidated, this IL-17 increment might not promote adhesion.

In summary, we have shown that PAI-1 and IL-17 may be key molecules for surgery-induced adhesion formation. Collagen deposition and leukocyte accumulation are histological features of postsurgical adhesion formation. DKT treatment did not have any inhibitory effect on adhesion formation in 2 mouse adhesion models.

Acknowledgments This work was supported by Grant-in-
Aid for special research promotion, Hyogo College of Medicine, 2014-2018. We are grateful to K. Mitani for technical help with immunohistochemistry.

Conflict of Interest The authors declare no conflict of interest.

Supplementary Materials The online version of this article contains supplementary materials.

\section{REFERENCES}

1) Menzies D, Ellis H. Intestinal-obstruction from adhesions-How big is the problem. Ann. Roy. Coll. Surg., 72, 60-63 (1990).

2) Cheong YC, Laird SM, Li TC, Shelton JB, Ledger WL, Cooke ID. Peritoneal healing and adhesion formation/reformation. Hum. Reprod. Update, 7, 556-566 (2001).

3) Diamond MP, Freeman ML. Clinical implications of postsurgical adhesions. Hum. Reprod. Update, 7, 567-576 (2001).

4) Vrijland WW, Jeekel J, van Geldorp HJ, Swank DJ, Bonjer HJ. Abdominal adhesions: intestinal obstruction, pain, and infertility. Surg. Endosc., 17, 1017-1022 (2003).

5) Demco L. Pain mapping of adhesions. J. Am. Assoc. Gynecol. Laparosc., 11, 181-183 (2004).

6) Sikirica V, Bapat B, Candrilli SD, Davis KL, Wilson M, Johns A. The inpatient burden of abdominal and gynecological adhesiolysis in the US. BMC Surg., 11, 13 (2011).

7) ten Broek RPG, Strik C, Issa Y, Bleichrodt RP, van Goor H. Adhesiolysis-related morbidity in abdominal surgery. Ann. Surg., 258 , 98-106 (2013).

8) Kosaka H, Yoshimoto $T$, Yoshimoto T, Fujimoto J, Nakanishi K. Interferon-gamma is a therapeutic target molecule for prevention of postoperative adhesion formation. Nat. Med., 14, 437-441 (2008).

9) Ohashi K, Yoshimoto T, Kosaka H, Hirano T, Iimuro Y, Nakanishi $\mathrm{K}$, Fujimoto J. Interferon gamma and plasminogen activator inhibitor 1 regulate adhesion formation after partial hepatectomy. $\mathrm{Br} . \mathrm{J}$. Surg., 101, 398-407 (2014).

10) Chung DR, Chitnis T, Panzo RJ, Kasper DL, Sayegh MH, Tzianabos AO. CD4+ T cells regulate surgical and postinfectious adhesion formation. J. Exp. Med., 195, 1471-1478 (2002).

11) Wang G, Wu K, Li W, Zhao ED, Shi L, Wang JL, Shuai XM, Cai KL, Lu XM, Tao KX, Wang GB. Role of IL-17 and TGF-beta in peritoneal adhesion formation after surgical trauma. Wound Repair Regen., 22, 631-639 (2014).

12) Reed KL, Lehrmann JF, Stucchi AF, Gower AC, Leeman SE, Becker JM. Administration of a substance $p$ receptor antagonist (SPRA) increases the expression of peritoneal tissue plasminogen activator (tPA) in a rat model of intraabdominal adhesions. Gastroenterology, 124, A299-A299 (2003).

13) Reed KL, Fruin AB, Bishop-Bartolomei KK, Gower AC, Nicolaou M, Stucchi AF, Leeman SE, Becker JM. Neurokinin-1 receptor and substance P messenger RNA levels increase during intraabdominal adhesion formation. J. Surg. Res., 108, 165-172 (2002).

14) Reed KL, Fruin AB, Gower AC, Stucchi AF, Leeman SE, Becker JM. A neurokinin 1 receptor antagonist decreases postoperative peritoneal adhesion formation and increases peritoneal fibrinolytic activity. Proc. Natl. Acad. Sci. U.S.A., 101, 9115-9120 (2004).

15) Esposito AJ, Heydrick SJ, Cassidy MR, Gallant J, Stucchi AF, Becker JM. Substance P is an early mediator of peritoneal fibrinolytic pathway genes and promotes intra-abdominal adhesion formation. J. Surg. Res., 181, 25-31 (2013).

16) Okada K, Kawai M, Hirono S, Miyazawa M, Shimizu A, Kitahata Y, Yamaue H. Perioperative administration of Daikenchuto (TJ-100) reduces the postoperative paralytic ileus in patients with pancreaticoduodenectomy. Hepatogastroenterology, 62, 466-471 (2015). 
17) Bauer AJ, Boeckxstaens GE. Mechanisms of postoperative ileus. Neurogastroenterol. Motil., 16 (Suppl. 2), 54-60 (2004).

18) Jin XL, Shibata C, Naito H, Ueno T, Funayama Y, Fukushima K, Matsuno S, Sasaki I. Intraduodenal and intrajejunal administration of the herbal medicine, dai-kenchu-tou, stimulates small intestinal motility via cholinergic receptors in conscious dogs. Dig. Dis. Sci., 46, 1171-1176 (2001).

19) Endo M, Hori M, Ozaki H, Oikawa T, Hanawa T. Daikenchuto, a traditional Japanese herbal medicine, ameliorates postoperative ileus by anti-inflammatory action through nicotinic acetylcholine receptors. J. Gastroenterol., 49, 1026-1039 (2014).

20) The FO, Boeckxstaens GE, Snoek SA, Cash JL, Bennink R, Larosa GJ, van den Wijngaard RM, Greaves DR, de Jonge WJ. Activation of the cholinergic anti-inflammatory pathway ameliorates postoperative ileus in mice. Gastroenterology, 133, 1219-1228 (2007).

21) Tracey KJ. Reflex control of immunity. Nat. Rev. Immunol., 9, 418-428 (2009).

22) Tokita $Y$, Yamamoto $M$, Satoh $K$, Nishiyama M, Iizuka S, Imamura $\mathrm{S}$, Kase Y. Possible involvement of the transient receptor potential vanilloid type 1 channel in postoperative adhesive obstruction and its prevention by a kampo (traditional Japanese) medicine, daikenchuto. J. Pharmacol. Sci., 115, 75-83 (2011).

23) Kono T, Shimada M, Yamamoto M, Kaneko A, Oomiya Y, Kubota K, Kase Y, Lee K, Uezono Y. Complementary and synergistic therapeutic effects of compounds found in Kampo medicine: analysis of daikenchuto. Front Pharmacol, 6, 159 (2015).

24) Krause TJ, Goldsmith NK, Ebner S, Zazanis GA, McKinnon RD. An inhibitor of cell proliferation associated with adhesion formation is suppressed by $N, O$-carboxymethyl chitosan. J. Invest. Surg., 11, 105-113 (1998).

25) Livak KJ, Schmittgen TD. Analysis of relative gene expression data using real-time quantitative PCR and the 2(T)(-Delta Delta C) method. Methods, 25, 402-408 (2001).

26) Kitamura M, Nishino T, Obata Y, Oka S, Abe S, Muta K, Ozono Y, Koji T, Kohno S. The kampo medicine Daikenchuto inhibits peritoneal fibrosis in mice. Biol. Pharm. Bull., 38, 193-200 (2015).

27) Kono T, Kaneko A, Hira Y, Suzuki T, Chisato N, Ohtake N, Miura $\mathrm{N}$, Watanabe T. Anti-colitis and -adhesion effects of daikenchuto via endogenous adrenomedullin enhancement in Crohn's disease mouse model. J. Crohns Colitis, 4, 161-170 (2010).

28) Jin XL, Shibata C, Naito H, Ueno T, Funayama Y, Fukushima K, Matsuno S, Sasaki I. Intraduodenal and intrajejunal administration of the herbal medicine, dai-kenchu-tou, stimulates small intestinal motility via cholinergic receptors in conscious dogs. Dig. Dis. Sci., 46, 1171-1176 (2001).

29) Endo M, Hori M, Ozaki H, Oikawa T, Hanawa T. Daikenchuto, a traditional Japanese herbal medicine, ameliorates postoperative ileus by anti-inflammatory action through nicotinic acetylcholine receptors. J. Gastroenterol., 49, 1026-1039 (2014).

30) Binder BR, Christ G, Gruber F, Grubic N, Hufnagl P, Krebs M, Mihaly J, Prager GW. Plasminogen activator inhibitor 1: physiological and pathophysiological roles. News Physiol. Sci., 17, 56-61 (2002).

31) Zorio E, Gilabert-Estelles J, Espana F, Ramon LA, Cosin R, Estelles A. Fibrinolysis: the key to new pathogenetic mechanisms. Curr. Med. Chem., 15, 923-929 (2008).

32) Loskutoff DJ, van Mourik JA, Erickson LA, Lawrence D. Detection of an unusually stable fibrinolytic inhibitor produced by bovine endothelial cells. Proc. Natl. Acad. Sci. U.S.A., 80, 2956-2960 (1983).

33) Loskutoff DJ, Quigley JP. PAI-1, fibrosis, and the elusive provi-

sional fibrin matrix. J. Clin. Invest., 106, 1441-1443 (2000).

34) Vipond MN, Whawell SA, Thompson JN, Dudley HA. Peritoneal fibrinolytic activity and intra-abdominal adhesions. Lancet, 335, 1120-1122 (1990).

35) Wang H, Zhang Y, Heuckeroth RO. PAI-1 deficiency reduces liver fibrosis after bile duct ligation in mice through activation of tPA. FEBS Lett., 581, 3098-3104 (2007).

36) Oda T, Jung YO, Kim HS, Cai XH, Lopez-Guisa JM, Ikeda Y, Eddy AA. PAI-1 deficiency attenuates the fibrogenic response to ureteral obstruction. Kidney Int., 60, 587-596 (2001).

37) Onishi RM, Gaffen SL. Interleukin-17 and its target genes: mechanisms of interleukin-17 function in disease. Immunology, 129, 311-321 (2010).

38) Taylor PR, Roy S, Leal SM Jr, Sun Y, Howell SJ, Cobb BA, Li XX, Pearlman E. Activation of neutrophils by autocrine IL-17A-IL-17RC interactions during fungal infection is regulated by IL-6, IL-23, ROR gamma t and dectin-2. Nat. Immunol., 15, 143-151 (2014).

39) Kelly MN, Kolls JK, Happel K, Schwartzman JD, Schwarzenberger P, Combe C, Moretto M, Khan IA. Interleukin-17/interleukin-17 receptor-mediated signaling is important for generation of an optimal polymorphonuclear response against Toxoplasma gondii infection. Infect. Immun., 73, 617-621 (2005).

40) Wu Q, Martin RJ, Rino JG, Breed R, Torres RM, Chu HW. IL23-dependent IL-17 production is essential in neutrophil recruitment and activity in mouse lung defense against respiratory Mycoplasma pneumoniae infection. Microbes Infect., 9, 78-86 (2007).

41) Hasan SA, Eksteen B, Reid D, Paine HV, Alansary A, Johannson K, Gwozd C, Goring KAR, Vo T, Proud D, Kelly MM. Role of IL-17A and neutrophils in fibrosis in experimental hypersensitivity pneumonitis. J. Allergy Clin. Immunol., 131, 1663-1673.e5 (2013).

42) Mehrotra P, Collett JA, McKinney SD, Stevens J, Ivancic CM, Basile DP. Inflammation and Inflammatory Mediators in Kidney Disease IL-17 mediates neutrophil infiltration and renal fibrosis following recovery from ischemia reperfusion: compensatory role of natural killer cells in athymic rats. Am. J. Physiol. Renal. Physiol., 312, F385-F397 (2017).

43) Wang G, Wu K, Li W, Zhao E, Shi L, Wang J, Shuai X, Cai K, Lu X, Tao K, Wang G. Role of IL-17 and TGF-beta in peritoneal adhesion formation after surgical trauma. Wound Repair Regen., 22, 631-639 (2014).

44) Thaler K, Mack JA, Berho M, Grotendorst G, Wexner SD, Abramson SR. Coincidence of connective tissue growth factor expression with fibrosis and angiogenesis in postoperative peritoneal adhesion formation. Eur. Surg. Res., 37, 235-241 (2005).

45) Inoue $\mathrm{K}$, Naito $\mathrm{Y}$, Takagi $\mathrm{T}$, Hayashi N, Hirai Y, Mizushima K, Horie R, Fukumoto K, Yamada S, Harusato A, Hirata I, Omatsu T, Yoshida N, Uchiyama K, Ishikawa T, Handa O, Konishi H, Wakabayashi N, Yagi N, Ichikawa H, Kokura S, Yoshikawa T. Daikenchuto, a Kampo medicine, regulates intestinal fibrosis associated with decreasing expression of heat shock protein 47 and collagen content in a rat colitis model. Biol. Pharm. Bull., 34, 1659-1665 (2011).

46) Nakai A, Satoh M, Hirayoshi K, Nagata K. Involvement of the Stress Protein Hsp47 in Procollagen Processing in the EndoplasmicReticulum. J. Cell Biol., 117, 903-914 (1992).

47) Canty EG, Kadler KE. Procollagen trafficking, processing and fibrillogenesis. J. Cell Sci., 118, 1341-1353 (2005).

48) Saed GM, Zhang W, Chegini N, Holmdahl L, Diamond MP. Alteration of type I and III collagen expression in human peritoneal mesothelial cells in response to hypoxia and transforming growth factor-beta 1. Wound Repair Regen., 7, 504-510 (1999).

49) Saed GM, Diamond MP. Hypoxia-induced irreversible up-regulation of type I collagen and transforming growth factor-beta 1 in human peritoneal fibroblasts. Fertil. Steril., 78, 144-147 (2002).

50) Saed GM, Zhang W, Diamond MP. Molecular characterization of fibroblasts isolated from human peritoneum and adhesions. Fertil. Steril., 75, 763-768 (2001). 